\title{
Neutrino Mass Matrix and Hierarchy
}

\author{
Peter Kaus \\ Physics Department, University of California, Riverside, CA 92521 \\ and \\ Sydney Meshkov \\ California Institute of Technology, Pasadena, CA 91125
}

\begin{abstract}
We build a model to describe neutrinos based on strict hierarchy, incorporating as much as possible, the latest known data, for $\Delta_{\text {sol }}$ and $\Delta_{a t m}$, and for the mixing angles determined from neutrino oscillation experiments, including that from KamLAND. Since the hierarchy assumption is a statement about mass ratios, it lets us obtain all three neutrino masses. We obtain a mass matrix, $M_{\nu}$ and a mixing matrix, $U$, where both $M_{\nu}$ and $U$ are given in terms of powers of $\Lambda$, the analog of the Cabibbo angle $\lambda$ in the Wolfenstein representation, and two parameters, $\rho$ and $\kappa$, each of order one. The expansion parameter, $\Lambda$, is defined by $\Lambda^{2}=m_{2} / m_{3}=\sqrt{ }\left(\Delta_{\text {sol }} / \Delta_{\text {atm }}\right) \approx 0.16$, and $\rho$ expresses our ignorance of the lightest neutrino mass $m_{1},\left(m_{1}=\rho \Lambda^{4} m_{3}\right)$, while $\kappa$ scales $s_{13}$ to the experimental upper limit, $s_{13}=\kappa \Lambda^{2} \approx 0.16 \kappa$. These matrices are similar in structure to those for the quark and lepton families, but with $\Lambda$ about 1.6 times larger than the $\lambda$ for the quarks and charged leptons. The upper limit for the effective neutrino mass in double $\beta$-decay experiments is $4 \times 10^{-3} \mathrm{eV}$ if $s_{13}=0$ and $6 \times 10^{-3} \mathrm{eV}$ if $s_{13}$ is maximal. The model, which is fairly unique, given the hierarchy assumption and the data, is compared to supersymmetric extension and texture zero models of mass generation.
\end{abstract}

\section{INTRODUCTION}

The hierarchical model has been very successful in describing the mass patterns and mixing matrices for quarks and charged leptons [1]. Both the mass patterns and mixing angles are dominated by an expansion parameter, which for each family is given by $\lambda=\sqrt{ }\left(m_{2} / m_{3}\right)$. Furthermore the $\lambda$ 's for the three families are roughly equal, $0.22<\lambda<0.25$. Here, we will try to see whether neutrinos can be brought simply into the standard fold. We have a fair handle on the mixing matrix, but as far as the masses are concerned, we only know the two mass-squared differences, $\Delta_{\text {sol }}$ and $\Delta_{a t m}$. This allows the mass ratio $\left(m_{2} / m_{3}\right)$ to range from about 1 (degeneracy) to small, $\sim 0.1$, (hierarchy). To determine all three masses one more equation is needed and it is provided by the hierarchy assumption.

CP672, Short Distance Behavior of Fundamental Interactions, edited by B. N. Kursunoglu et al. (C) 2003 American Institute of Physics 0-7354-0139-X/03/\$20.00 
The three neutrino mass eigenvalues, $m_{1}, m_{2}, m_{3}$ give a diagonal mass matrix. This matrix can be undiagonalised by the mixing matrix, $U$, and the results classified [2] according to possible mass assignments consistent with $\Delta_{\text {sol }}$ and $\Delta_{\text {atm }}$. In this paper, we try to build a model based on strict hierarchy, incorporating the known data as much as possible. Since the hierarchy assumption is a statement about mass ratios, it lets us obtain all three masses from the two $\Delta$ 's. This leads to a mass matrix and mixing matrix, almost entirely in terms of powers of $\Lambda$, the ana$\log$ of the Cabibbo angle $\lambda$ in the Wolfenstein representation. These matrices are similar in structure to the quark and lepton families, but with $\Lambda$ about 1.6 times larger than $\lambda$ for the other families. The effective mass, $\langle m\rangle$, measured in $\beta \beta_{0 \nu}$ decay [3], is then obtained. The model, which is fairly unique given the hierarchy assumption and the data, is compared to models of mass generation $[4,5]$.

\section{DETERMINATION OF THE NEUTRINO MASS MATRIX AND MIXING MATRIX}

The mixing matrix, $U,[6]$ which rotates mass (Majorana) eigenstates $\Psi_{1,2,3}$ into flavor eigenstates $\Psi_{\nu_{e}, \nu_{\mu}, \nu_{\tau}}$ is parameterized as usual

$$
U=\left[\begin{array}{ccc}
c_{12} c_{13} & -s_{12} c_{13} & s_{13} e^{-i \partial} \\
s_{12} c_{23}+c_{12} s_{13} s_{23} e^{i \partial} & c_{12} c_{23}-s_{12} s_{13} s_{23} e^{i \partial} & -c_{13} s_{23} \\
s_{12} s_{23}-c_{12} s_{13} c_{23} e^{i \partial} & c_{12} s_{23}+s_{12} s_{13} c_{23} e^{i \partial} & c_{13} c_{23}
\end{array}\right]
$$

Letting $\delta=0$ (no $\mathrm{CP}$ violation), and assuming maximal mixing for the atmospheric oscillation [7], i.e., $s_{23}=c_{23}=1 / \sqrt{ } 2$, we have for $U$ :

$$
U=\left[\begin{array}{ccc}
C c_{13} & -S c_{13} & s_{13} \\
\frac{1}{\sqrt{2}} S+\frac{1}{\sqrt{2}} C s_{13} & \frac{1}{\sqrt{2}} C-\frac{1}{\sqrt{2}} S s_{13} & -\frac{1}{\sqrt{2}} c_{13} \\
\frac{1}{\sqrt{2}} S-\frac{1}{\sqrt{2}} C s_{13} & \frac{1}{\sqrt{2}} C+\frac{1}{\sqrt{2}} S s_{13} & \frac{1}{\sqrt{2}} c_{13}
\end{array}\right]
$$

The angle $\theta_{13}$ is known to be small $[8,9,10]$, with an upper limit $s_{13}<0.13$ and at present no lower limit. We start with the two rotations known not to vanish, $\theta_{12}$ and $\theta_{23}$. With $\theta_{13}=0$, we have:

$$
U=\left[\begin{array}{ccc}
C & -S & 0 \\
S / \sqrt{2} & C / \sqrt{2} & -1 / \sqrt{2} \\
S / \sqrt{2} & C / \sqrt{2} & 1 / \sqrt{2}
\end{array}\right]
$$

where $S=\sin \left(\theta_{12}\right), C=\cos \left(\theta_{12}\right)$ and we have set the rotation angle $\theta_{23}=\pi / 4$ (maximal mixing) and the angle $\theta_{13}=0$, no $\mathrm{CP}$ violation. 
To lowest order in $S$ (expanding $C$ in Eq. (3) in terms of $S$ ), $U$ is given by

$$
U_{1}=\left[\begin{array}{ccc}
1 & -S & 0 \\
S / \sqrt{2} & 1 / \sqrt{2} & -1 / \sqrt{2} \\
S / \sqrt{2} & 1 / \sqrt{2} & 1 / \sqrt{2}
\end{array}\right]
$$

which, except for the extreme $\theta_{23}$ mixing, is much like the quark mixing matrix. In fact, if we consider $U_{1}$ to be the result of two successive rotations, $U_{1}=v_{1} v_{0}$, we get

$$
v_{0}=\left[\begin{array}{ccc}
1 & 0 & 0 \\
0 & 1 / \sqrt{2} & -1 / \sqrt{2} \\
0 & 1 / \sqrt{2} & 1 / \sqrt{2}
\end{array}\right]
$$

and

$$
v_{1}=\left[\begin{array}{ccc}
1 & -S / \sqrt{2} & -S / \sqrt{2} \\
S / \sqrt{2} & 1 & 0 \\
S / \sqrt{2} & 0 & 1
\end{array}\right]
$$

This suggests that the appropriate expansion parameter for $U$ is given by:

$$
\varepsilon=S / \sqrt{ } 2=\sin \left(\theta_{12}\right) / \sqrt{ } 2 .
$$

Recent KamLAND experiments [11] and analyses of this data $[12,13,14]$, extracting $\theta_{12}$, show that it is roughly $34^{\circ}$, i.e., $\tan ^{2}\left(\theta_{12}\right) \approx 0.45$, consistent with earlier SNO experiments [15].

Turning to the diagonal mass matrix, we define the conventional hierarchical mass pattern by:

$$
m_{3}: m_{2}: m_{1}=1: \Lambda^{2}: \rho \Lambda^{4} .
$$

( $\rho=1$ would correspond to strict hierarchy). The three eigenvalues are then $m_{3}, m_{2}=\Lambda^{2} m_{3}$ and $m_{1}=\rho \Lambda^{4} m_{3}$.

The hierarchy expansion is in terms of the traditional hierarchy parameter, $\Lambda$.

$$
\Lambda=\sqrt{ }\left(m_{2} / m_{3}\right) .
$$

For quarks and charged leptons, the mass matrices, parametrized by $\lambda$, and the mixing matrices, given in terms of $\theta_{i j}$ are related. The observed mixing angles of the mixing matrices are given as powers of $\lambda$, the Cabibbo angle, as seen in the Wolfenstein [16] representation of the $V_{C K M}$. We will try to determine the analogous relationship for neutrinos from the data. We note that both expansion parameters, $\varepsilon$ and $\Lambda$, can be evaluated, independently, from experimental data. With $\tan ^{2}\left(\theta_{12}\right)=T^{2} \approx 0.45$, we have:

$$
S^{2} \approx 0.31
$$


and therefore

$$
\varepsilon^{2}=S^{2} / 2 \approx 0.16
$$

On the other hand $\Lambda^{2}=m_{2} / m_{3}$, (the expansion parameter for the mass matrix) can be evaluated in the hierarchical expansion, using

$$
\Delta_{\text {sol }}=m_{2}^{2}-m_{1}^{2} \approx 7.1 \times 10^{-5} \mathrm{eV}^{2}
$$

and

$$
\Delta_{a t m}=m_{3}^{2}-m_{2}^{2} \approx 2.7 \times 10^{-3} \mathrm{eV}^{2}
$$

which forms the ratio

$$
\begin{gathered}
\sqrt{ }\left(\Delta_{\text {sol }} / \Delta_{a t m}\right)=\sqrt{ }\left[\left(m_{2}^{2}-m_{1}^{2}\right) /\left(m_{3}^{2}-m_{2}^{2}\right)\right] \\
=\sqrt{ }\left[\left(\Lambda^{4} m_{3}^{2}-\rho^{2} \Lambda^{8} m_{3}^{2}\right) /\left(m_{3}^{2}-\Lambda^{4} m_{3}^{2}\right)\right] .
\end{gathered}
$$

The value for $\Delta_{\text {sol }}$ is obtained in the analyses of $\operatorname{KamLAND}$ data $[12,13]$; the value for $\Delta_{a t m}$ is obtained from analyses by S. Pascoli, et al., [14] and G.L. Fogli, et al., [17]. Expanding in $\Lambda^{2}$ we obtain

$$
\sqrt{ }\left(\Delta_{s o l} / \Delta_{a t m}\right)=\Lambda^{2}+(1 / 2) \Lambda^{6}\left(1-\rho^{2}\right) .
$$

Thus to order $\Lambda^{4}$ we have

$$
\Lambda^{2}=\sqrt{ }\left(\Delta_{\text {sol }} / \Delta_{\text {atm }}\right)=0.16
$$

so that

$$
\varepsilon^{2}=\Lambda^{2}=0.16
$$

or

$$
\sin \left(\theta_{12}\right) / \sqrt{ } 2 \approx \Lambda
$$

Phenomenologically, at least, there is a close relationship between $\theta_{12}$ and $\Lambda$.

We will use equation 16 as an equality. We can now express both the mass matrix, $M$, as well as the rotation matrix $U$, in terms of one parameter, defined in equation 7 as $\Lambda=\sqrt{ }\left(m_{2} / m_{3}\right)$, in analogy with the Cabibbo angle for quarks and charged leptons.

The mixing matrix, $U$, is now

$$
U=\left[\begin{array}{ccc}
\sqrt{\left(1-2 \Lambda^{2}\right)} & -\sqrt{2} \Lambda & 0 \\
\Lambda & \sqrt{(1 / 2)\left(1-2 \Lambda^{2}\right)} & -1 / \sqrt{2} \\
\Lambda & \sqrt{(1 / 2)\left(1-2 \Lambda^{2}\right)} & -1 / \sqrt{2}
\end{array}\right]
$$

While $\Lambda=0.4$ is a rather large number, the expansions made in equation 17 are of the square roots and thus, effectively, the expansion parameter is $\Lambda^{2}=0.16$. 
The mass matrix $M_{\nu}$ is given by

$$
M_{\nu}=U M U^{-1}
$$

with

$$
M=m_{3}\left[\begin{array}{ccc}
\Lambda^{4} \rho & 0 & 0 \\
0 & \Lambda^{2} & 0 \\
0 & 0 & 1
\end{array}\right]
$$

To order $\Lambda^{4}, M_{\nu}$, is given by

$$
M_{\nu_{4}}=m_{3}\left[\begin{array}{ccc}
\Lambda^{4}(\rho+2) & -\Lambda^{3} & -\Lambda^{3} \\
-\Lambda^{3} & -\Lambda^{4}+(1 / 2) \Lambda^{2}+1 / 2 & -\Lambda^{4}+(1 / 2) \Lambda^{2}-1 / 2 \\
-\Lambda^{3} & -\Lambda^{4}+(1 / 2) \Lambda^{2}-1 / 2 & -\Lambda^{4}+(1 / 2) \Lambda^{2}+1 / 2
\end{array}\right]
$$

where, using eq. (7), $\Lambda=\sqrt{ }\left(m_{2} / m_{3}\right)=\sqrt{ } \cdot 16=0.4$ and

$$
m_{3}=\sqrt{ }\left(\Delta_{\text {atm }}+\Delta_{\text {sol }}\right)=5.2 \times 10^{-2} \mathrm{eV}
$$

While the analytic expressions are to the orders in $\Lambda$ indicated, the data, unfortunately, are not. Thus, all results will be given to at most two significant figures.

We now can determine the masses, $m_{1}, m_{2}$ and $m_{3}$ to order $\Lambda^{4}$ :

$$
\begin{gathered}
m_{3}=\sqrt{ }\left(\Delta_{a t m}+\Delta_{s o l}\right)=5.2 \times 10^{-2} \mathrm{eV} \\
m_{2}=\Lambda^{2} m_{3}=\sqrt{ }\left(\Delta_{s o l} / \Delta_{a t m}\right) m_{3}=8.3 \times 10^{-3} \mathrm{eV}
\end{gathered}
$$

and

$$
m_{1}=\rho \Lambda^{4} m_{3}=\rho\left(\Delta_{\text {sol }} / \Delta_{\text {atm }}\right) m_{3}=1.3 \rho \times 10^{-3} \mathrm{eV}
$$

We may let $\rho$ range from, say, 2 to -2 and still consider $\left|m_{1}\right|$ to be of order $\Lambda^{4}$ or smaller. These masses follow directly from the hierarchy assumption and the experimental values: $\Delta_{\text {sol }}=m_{2}^{2}-m_{1}^{2} \approx 7.1 \times 10^{-5} \mathrm{eV}^{2}$ and $\Delta_{a t m}=m_{3}^{2}-m_{2}^{2} \approx$ $2.7 \times 10^{-3} \mathrm{eV}^{2}$ and are independent of the mixing matrix $U$. The effective neutrino mass $\left\langle m>\right.$ measured in $\beta \beta_{0 \nu}$ decay, [3] however, does depend on the neutrino mass matrix, $M_{\nu}$ and is given by $M_{\nu_{e e}}$, the 1,1 matrix element. From equations (17) and (19) we have, to order $\Lambda^{6}$

$$
<m>=M_{\nu_{e e}}=m_{3} \Lambda^{4}\left[\rho\left(1-2 \Lambda^{2}\right)+2\right]=(0.7 \rho+2) \times 1.3 \times 10^{-3} \mathrm{eV}
$$

Taking $\rho=2,\left(m_{1}=2.6 \times 10^{-3} \mathrm{eV}\right)$ as an extreme case, we obtain as an uper limit (with $s_{13}=0$ ):

$$
<m>\leq 4 \times 10^{-3} \mathrm{eV}
$$

On the other hand, $m_{1}$ and therefore $\rho$, may be negative. For $\rho=-2,\left(m_{1}=\right.$ $\left.-2.6 \times 10^{-3} \mathrm{eV}\right)$, we have

$$
\langle m\rangle=M_{\nu_{e e}}=\Lambda^{6} m_{3}=8 \times 10^{-4} \mathrm{eV} .
$$

Thus, the limits on $\langle m\rangle$ in this model are, approximately,

$$
10^{-3}<<m><4 \times 10^{-3} \mathrm{eV}
$$




\section{INCLUSION OF $s_{13}=\sin \left(\theta_{13}\right)$}

While the present data do not demand a non-vanishing $\theta_{13}$, several models of hierarchy generation do $[2,18]$. We want to investigate the effect of a finite $s_{13}$.

Since we know that $s_{13}<0.13[8,9,10]$ and there is at present no lower limit, we will scale $s_{13}$ :

$$
s_{13}=\kappa \Lambda^{2}
$$

where $1 \geq|\kappa| \geq 0$.

Substituting for $s_{13}$ in eq. (2) and forming the mass matrix $M_{\nu}$, we obtain to order $\Lambda^{4}$ :

$M_{\nu_{4}}=m_{3}\left[\begin{array}{ccc}\Lambda^{4}\left(\kappa^{2}+\rho+2\right) & -\Lambda^{3}-\frac{1}{\sqrt{2}} \Lambda^{2} \kappa & -\Lambda^{3}+\frac{1}{\sqrt{2}} \Lambda^{2} \kappa \\ -\Lambda^{3}-\frac{1}{\sqrt{2}} \Lambda^{2} \kappa & -\frac{1}{2} \Lambda^{4}\left(\kappa^{2}+1\right)+\frac{1}{2} \Lambda^{2}+\frac{1}{2} & \frac{1}{2} \Lambda^{4}\left(\kappa^{2}+1\right)+\frac{1}{2} \Lambda^{2}-\frac{1}{2} \\ -\Lambda^{3}+\frac{1}{\sqrt{2}} \Lambda^{2} \kappa & \frac{1}{2} \Lambda^{4}\left(\kappa^{2}-1\right)+\frac{1}{2} \Lambda^{2}-\frac{1}{2} & -\frac{1}{2} \Lambda^{4}\left(\kappa^{2}+1\right)+\frac{1}{2} \Lambda^{2}+\frac{1}{2}\end{array}\right]$

We now have two parameters, $\rho$ and $\kappa$, where $\rho$ is defined by $m_{1}=\rho \Lambda^{4} m_{3}$ and $s_{13}=\kappa \Lambda^{2},|\kappa| \leq 1$. There are three special regimes for $\kappa$ which are interesting.

1. $\kappa=0, s_{13}=0$ This is the case which was discussed earlier. We give here the leading elements of $M_{\nu}$ which depend on $\kappa$.

$$
\begin{gathered}
M_{\nu_{e e}}=<m>=\Lambda^{4}(\rho+2) m_{3} \\
M_{\nu_{e \mu}}=M_{\nu_{e 3}}=-\Lambda^{3} m_{3}
\end{gathered}
$$

2. $\kappa \approx 1$ (upper limit), $s_{13}=\Lambda^{2}$

$$
\begin{gathered}
M_{\nu_{e e}=<} m>=\Lambda^{4}\left(\rho+2+\kappa^{2}\right) m_{3}=\Lambda^{4}(\rho+3) m_{3} \\
M_{\nu_{e \mu}}=-M_{\nu_{e \tau}}= \pm(1 / \sqrt{ } 2) \Lambda^{2} m_{3}
\end{gathered}
$$

and the most interesting possibility,

3. $\kappa=\kappa^{\prime} \Lambda$ i.e., $s_{13}=\kappa^{\prime} \Lambda^{3}$. For case (3) the mass matrix to order $\Lambda^{4}$ is:

$$
M_{\nu_{4}}=m_{3}\left[\begin{array}{ccc}
\Lambda^{4}(\rho+2) & -\Lambda^{3}\left(1+\frac{\kappa^{\prime}}{\sqrt{2}}\right) & -\Lambda^{3}\left(1-\frac{\kappa^{\prime}}{\sqrt{2}}\right) \\
-\Lambda^{3}\left(1+\frac{\kappa^{\prime}}{\sqrt{2}}\right) & -\Lambda^{4}+\Lambda^{2} / 2+\frac{1}{2} & -\Lambda^{4}+\Lambda^{2} / 2-\frac{1}{2} \\
-\Lambda^{3}\left(1-\frac{\kappa^{\prime}}{\sqrt{2}}\right) & -\Lambda^{4}+\Lambda^{2} / 2-\frac{1}{2} & -\Lambda^{4}+\Lambda^{2} / 2+\frac{1}{2}
\end{array}\right]
$$

and

$$
\begin{aligned}
& M_{\nu_{e \mu}}=-\Lambda^{3}\left[1+(1 / \sqrt{ } 2) \kappa^{\prime}\right] m_{3} \\
& M_{\nu_{e \tau}}=-\Lambda^{3}\left[1-(1 / \sqrt{ } 2) \kappa^{\prime}\right] m_{3}
\end{aligned}
$$

Case (3) is the only case which allows a zero in an off diagonal element. Texture zeros have been considered as a possible source of hierarchies and mixing angles $[4$, 
$5,19,20]$. From Eq. (35), we see that only $\kappa=\kappa^{\prime} \Lambda$ provides the possibility of having two texture zeroes.

Taking $\rho=-2$ and $\kappa^{\prime}= \pm \sqrt{ } 2$ will make $M_{\nu_{e e}}$ and either $M_{\nu_{e \mu}}$ or $M_{\nu_{e r}}$ vanish to order $\Lambda^{4}$. With $\kappa^{\prime}=-\sqrt{ } 2, M_{\nu_{4}}$ becomes

$$
M_{\nu_{4}}=m_{3}\left[\begin{array}{ccc}
0 & 0 & -2 \Lambda^{3} \\
0 & -\Lambda^{4}+\Lambda^{2} / 2+\frac{1}{2} & -\Lambda^{4}+\Lambda^{2} / 2-\frac{1}{2} \\
-2 \Lambda^{3} & -\Lambda^{4}+\Lambda^{2} / 2-\frac{1}{2} & -\Lambda^{4}+\Lambda^{2} / 2+\frac{1}{2}
\end{array}\right]
$$

In terms of masses we substitute $m_{2}=\Lambda^{2} m_{3}, m_{1}=\rho \Lambda^{4} m_{3}=-2 \Lambda^{4} m_{3}$ and get

$$
M_{\nu_{4}}=\left[\begin{array}{ccc}
0 & 0 & -\sqrt{\left(-2 m_{1} m_{2}\right)} \\
0 & \frac{1}{2}\left(m_{1}+m_{2}+m_{3}\right) & \frac{1}{2}\left(m_{1}+m_{2}-m_{3}\right) \\
-\sqrt{\left(-2 m_{1} m_{2}\right)} & \frac{1}{2}\left(m_{1}+m_{2}-m_{3}\right) & \frac{1}{2}\left(m_{1}+m_{2}+m_{3}\right)
\end{array}\right]
$$

Eq. (39) is identical to the matrix derived for the hierarchical case by B. R. Desai et. al.[20], who systematically categorize the neutrino mass matrices, consistent with experimental constraints, with two texture zeros. Note that for this model $<m>=M_{\nu_{e e}}$ vanishes to order $\Lambda^{4}$. In order to compare with a recent model for hierarchy generation, [4] we continue with $\rho=-2$, but do not specify $\kappa^{\prime}$ in $s_{13}=\kappa^{\prime} \Lambda^{3}$. In that case, keeping only the leading order in $\Lambda$ in each matrix element of $M_{\nu_{6}}$, we obtain :

$$
M_{\nu_{6}}=m_{3}\left[\begin{array}{ccc}
\Lambda^{6}\left(4+\kappa^{\prime 2}\right) & -\Lambda^{3}\left(1+\frac{1}{\sqrt{2}} \kappa^{\prime}\right) & -\Lambda^{3}\left(1-\frac{1}{\sqrt{2}} \kappa^{\prime}\right) \\
-\Lambda^{3}\left(1+\frac{1}{\sqrt{2}} \kappa^{\prime}\right) & \frac{1}{2} & -\frac{1}{2} \\
-\Lambda^{3}\left(1-\frac{1}{\sqrt{2}} \kappa^{\prime}\right) & -\frac{1}{2} & \frac{1}{2}
\end{array}\right]
$$

The leading orders of $\Lambda$ in each matrix element are the orders indicated in the work of Ramond, et al., [4] (and 'tuned' by Fishbane and Kaus [21]). This model suggests , within a super symmetric extension of the standard model, that the existence of mass hierarchies within fermionic sectors imply at least one additional $U(1)$ family symmetry one of which must be anomalous, with a cancellation of its anomaly through the Green-Schwarz mechanism then implying relations across fermionic sectors. This has the additional property of predicting $\Lambda$, which should be the same for all families. However, the data for neutrinos, $\Delta_{a t m}$ and $\Delta_{\text {sol }}$, suggest that $\Lambda \approx 0.4$, while for the other family sectors, we have the traditional $\lambda \approx 0.25$.

\section{SUMMARY}

We have shown that the assumed hierarchy pattern and the present data imply that a mixing matrix, $U$, and mass matrix, $M_{\nu}$ may be expressed in terms of powers of the expansion parameter $\Lambda$ and two parameters $\rho$ and $\kappa$ of order 
one. The parameter $\rho$ expresses our ignorance of the lightest neutrino mass, $m_{1}$, where $m_{1}=\rho \Lambda^{4} m_{3} \approx 1.3 \rho \times 10^{-3} \mathrm{eV}$ and $\kappa$ scales $s_{13}$ to the experimental upper limit, $s_{13}=\kappa \Lambda^{2} \approx 0.16 \kappa$. The simplicity of $U$ and $M_{\nu}$ comes from the observed relationship, $S^{2} / 2=\Lambda^{2}=m_{2} / m_{3}$. The expansion parameter, $\Lambda$, where $\Lambda^{2}=m_{2} / m_{3}=\sqrt{ }\left(\Delta_{\text {sol }} / \Delta_{\text {atm }} \approx 0.16\right.$ is identical in spirit, though not in value, to the Wolfenstein parameter [16], $\lambda$, in the quark $V_{C K M}$ and is measured by solar and atmospheric oscillation experiments. The upper limit for the effective neutrino mass in double $\beta$-decay experiments is $4 \times 10^{-3} \mathrm{eV}$ if $s_{13}=0$ and $6 \times 10^{-3} \mathrm{eV}$ if $s_{13}$ is maximal.

The models of hierarchical mass generation that we compared to, supersymmetric extension [4] and texture zeroes $[4,5,19,20]$ each demand that $M_{\nu_{e e}}$ be of order $\Lambda^{6}$ or even vanish. This implies $\rho=-2, m_{1} \approx-2.6 \times 10^{-3} \mathrm{eV}$, in Eq. (30), and thus $\left\langle m>\approx 10^{-3} \mathrm{eV}\right.$ or smaller. Both of these models require the $M_{\nu_{e \mu}}$ and $M_{\nu_{e \tau}}$ terms to be of order $\Lambda^{3}$ or smaller. Therefore $s_{13}$ in Eq. (30) has to be of order $\Lambda^{3}$, i.e., $s_{13}=\kappa^{\prime} \Lambda^{3}=0.06 \kappa^{\prime}$, where $\kappa^{\prime}$ is of order unity or smaller. More specifically, for a texture zero in $M_{\nu_{e \mu}}$ or $M_{\nu_{e \tau}}$ one must have $\kappa^{\prime}= \pm \sqrt{ } 2$ or $s_{13} \approx 0.09$. All these demands are well below the present upper experimental upper limits of $\left\langle m>\right.$ and $s_{13}$.

\section{ACKNOWLEDGMENTS}

We thank the Aspen Center for Physics, where this work started, for its hospitality and stimulating atmosphere.

\section{REFERENCES}

[1] H. Fritzsch and Z. Xing, Prog. Part. Nucl. Phys., 45 (2000) 1, and Ref. 51 therein; P. Fishbane and P. Kaus, J. Phys., G26 (2000) 295.

[2] E. Ma, arXiv: hep-ph/0208097.

[3] S. Pascoli and S.T. Petcov, Phys. Lett., B544 (2002) 239.

[4] See, for example, F. S. Ling and P. Ramond, Phys. Lett., B543 (2002) 29; and earlier work, Irges, S. Lavignac and P. Ramond, Phys. Rev., D58 (1998) 035003.

[5] S. F. King, arXiv: hep-ph/0208266. 
[6] Z. Maki, M. Nakagawa, and S. Sakata, Prog. Theor. Phys., 28 (1962) 870; Super-Kamiokande Collaboration, S. Fukuda et al., Phys. Rev. Lett., 86 (2001) 5651-5656.

[7] S. Fukuda et. al., Phys. Rev. Lett., 85 (2000) 3999 and references therein.

[8] M. Appolonio et al., Phys. Lett., B 466 (1999) 415 (CHOOZ Collaboration.

[9] F. Boehm et al., Phys. Rev., D64 (2001) 112001 (PaloVerde Collaboration).

[10] G.L. Fogli, et al., arXiv: hep-ph/021247.

[11] K. Eguchi, et al., Phys. Rev. Lett., 90 (2003) 021802.

[12] J.N. Bahcall, M.C. Gonzales-Garcia and C. Pena-Garay, arXiv: hep$\mathrm{ph} / 0212147$.

[13] V. Barger and D. Marfatia, arXiv: hep-ph/0212126.

[14] S. Pascoli, S.T. Petcov and W. Rodejohann, arXiv: hep-ph/0212113.

[15] Q. R. Ahmed et al., Phys. Rev. Lett., 89 (2002) 011301, 011302.

[16] L. Wolfenstein, Phys. Rev. Lett., 51 (1983) 1945.

[17] G.L. Fogli, et al., arXiv: hep-ph/0208026.

[18] C. Giunti and M. Tanimoto, Phys. Rev, D66 (2002) 053013.

[19] P. Frampton, S. Glashow, and D. Marfatia, Phys. Lett., B536 (2002) 79.

[20] B. R. Desai, D. P. Roy, and A. R. Vaucher, arXiv: hep-ph/0209035.

[21] P. Fishbane and P. Kaus, J. Phys., G25 (1999) 1629. 